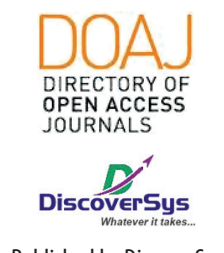

Published by DiscoverSys

\section{Jejunal atresia in the newborn: three cases after resection and end-to-end anastomoses}

\author{
Ni Made Sukewanti, ${ }^{1 *}$ I Wayan Dharma Artana, ${ }^{1}$ Putu Junara Putra, ${ }^{1}$ I Made \\ Kardana, ${ }^{1}$ Made Sukmawati, ${ }^{1}$ Kadek Deddy Ariyanta ${ }^{2}$
}

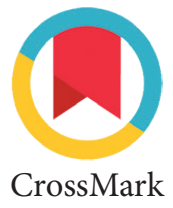

CrossMark

\begin{abstract}
Background: Intestinal atresia is a common cause of neonatal intestinal obstruction. Jejunal atresia occurs more frequent than duodenal or colonic atresias while single atresias are most commonly encountered. Jejunal atresia is classified into 4 types: Type I (a mucosal/ septal), Type II (a fibrous cord/band), Type III (blind ends are entirely separated without a fibrous cord between them and a large mesenteric defect), and Type IV (combination of atresia type I to III). This study aims to elaborate jejunal atresia in the newborn after resection and end-to-end anastomoses.
\end{abstract}

Case Description: We report three cases of jejunal atresia consists of different types: type IV (first case), type IIla (second case) and type I (third case). Cases were neonates born with signs of upper gut obstruction. Bile-stained vomiting was reported a few hours after birth and failed to pass meconium in the first 24 hours. Postnatal abdominal $X$-ray showed dilatation of the gaster and no gas present in the pelvic floor. In the third case, it showed a triple bubble sign on abdominal $x$-ray and a laparotomy revealed the type of jejuno-ileal atresia. Also, the resection on the atresias and end to end anastomosis were performed. Cases were admitted to neonatal intensive care unit with total parenteral nutrition. The first two cases died due to sepsis, while the third case survived.

Conclusion: Jejunal atresia is a rare disease among newborn. All of the patients have provided resection and end-to-end anastomoses, although 2 of them did not survive.
'Department of Child Health, Faculty of Medicine, Universitas Udayana, Sanglah General Hospital, Bali, Indonesia ${ }^{2}$ Pediatric Surgery, Faculty of Medicine, Universitas Udayana, Sanglah General Hospital, Bali, Indonesia
*Correspondence to: Ni Made Sukewanti; Department of Child Health, Faculty of Medicine Udayana University, Sanglah General Hospital, Bali, Indonesia; sukewantinimade@gmail.com

Received: 2019-06-19 Accepted: 2020-03-19 Published: 2020-03-26

Keywords: Jejunal Atresia, Newborn, Resection, Anastomoses.

Cite This Article: Sukewanti, N.M., Artana, I.W.D., Putra, P.J., Kardana, I.M., Sukmawati, M., Ariyanta, K.D. 2020. Jejunal atresia in the newborn: three cases after resection and end-to-end anastomoses. Intisari Sains Medis 11(1): 211-215. D0I: 10.15562/ism.v11i1.538

\section{INTRODUCTION}

Intestinal atresia is a congenital disorder characterized by complete occlusion of the bowel lumen. The incidence of jejuno-ileal atresia is $1-3$ per10,000 live births, while the incidence of duodenal atresia reaches 1 per 5,000-10,000 live births. ${ }^{1,2}$ Failure of recanalization and vascular accident or any other intestinal injuries are main approaches that explain the formation of atresia. ${ }^{3}$

About 6-12 percent of infants are diagnosed with multiple atretic segments worldwide. Jejunoileal atresia classified into 4 types. Type I is marked by a mucosal (septal) atresia, stenosis (a localized narrowing of the intestinal lumen without disruption of continuity or defect in mesentery) of the intestine., ${ }^{2,4}$ Type II is marked by a fibrous cord (band) separates both atretic segments, the mesentery is usually intact, and the length of the intestine is normal. Type III has two sub-divisions: type IIIa indicates both blind ends are entirely separated without a fibrous cord between them. The atresia has a V-shaped mesenteric gap, and the intestine is shortened. ${ }^{2,4}$ Type IIIb (apple peel or Christmas tree deformity) indicates both separated intestinal segments and a large mesenteric defect. Type IV means multiple atresias refers to any number and combination of atresias type I to III, and the intestinal length is always reduced often having the morphological appearance of sausage. ${ }^{2,4}$

Most newborns with an intestinal obstruction present with bilious vomiting which in the neonate should be considered secondary to a mechanical obstruction until proven otherwise. Emergency surgical evaluation is required in every newborn with this symptom. The role of diagnostic imaging is to help determine as accurately as possible the exact nature of the abnormality. ${ }^{5,6}$ Besides, the appropriate surgical procedure is necessarily important to improve the outcome and minimalize the complications. Based on those mentioned above, this case reports aim to evaluate the outcome of resection and end-to-end anastomoses surgical technique to the newborn with jejunal atresia.

\section{CASE DESCRIPTION}

\section{First Case}

A female newborn delivered at 34 weeks of gestation with cesarean section due to premature rupture of fetal membranes. Antenatal ultrasound revealed polyhydramnios on the third trimester and congenital megacolon. The patient was the third child in the family, no history of congenital anomalies in 
both parents. No illness history during the pregnancy period was noted, nor a history of consuming any traditional medicine as well.

She was born with a bodyweight of 2400 grams, body length of $45 \mathrm{~cm}$ and preterm in gestational age. There was no vomiting during the first hour. Mild abdominal distension was present and she was failed to pass meconium in the early 24 hours as well. There was a lot of orogastric tube production, approximately $50 \mathrm{cc}$, and it was bile-stained. The plain abdominal X-ray showed abdomen distention with intestinal dilatation, no double bubble sign, but there was no gas revealed in the pelvic floor.

Based on clinical manifestation, laboratory findings, and the plain abdominal X-ray, she was diagnosed with jejuno-ileal atresia. The patient was fasting. She was given total parenteral nutrition and planned to have explorative laparotomy surgery by a pediatric surgeon. On the third day, the patient was having laparotomy. On the surgery site, the surgeon found multiple atresias (type IV) (Figure 1a) on jejunum $35 \mathrm{~cm}$ from ligamentum Treitz and $110 \mathrm{~cm}$ from the ileocaecal junction. The

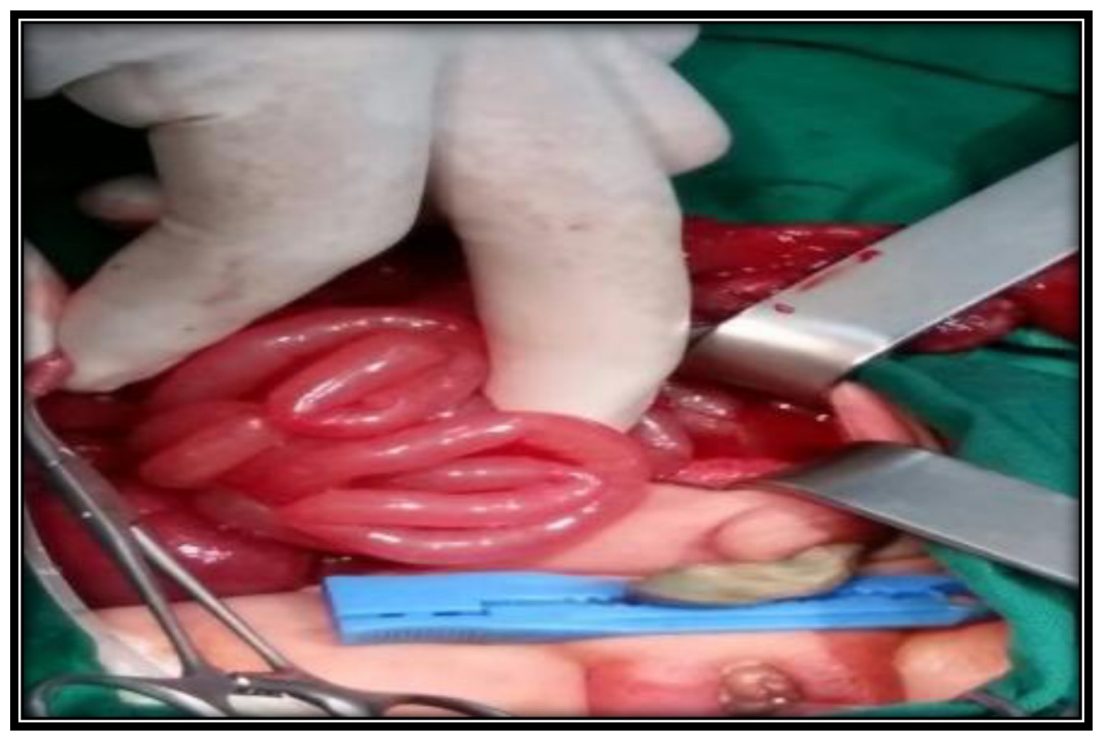

Figure 1 (a) Multiple jejunal atresia with Type IV and (b) End-to-end anastomoses surgical technique
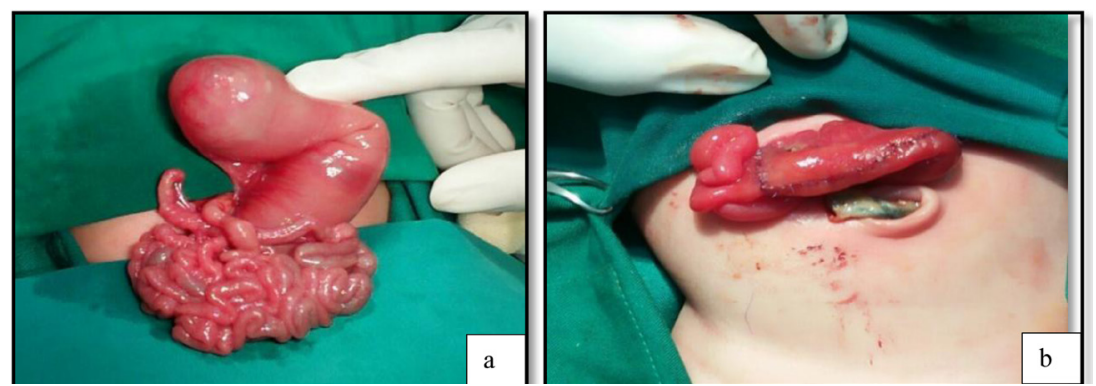

Figure 2 (a) Duodenum and proximal jejunum dilatation with jejuno-ileal atresia with type IIIa; (b) resection and continued with end-to-end anastomoses procedure patient had resection on jejuno-ileal atresia and continued with end to end anastomosis (Figure 1b).

The patient was delivered to the NICU after surgery. The patient was given total parenteral nutrition with orogastric tube decompression. The septic marker evaluation revealed: the leukocyte count was $17.3 \mathrm{~K} / \mathrm{uL}$, platelet count was $258 \mathrm{~K} / \mathrm{uL}$, IT ratio was 0.04, CRP 171.7. The patient was diagnosed with a suspect of early-onset neonatal sepsis and given intravenous antibiotic Ampicillin and Amikacin. After 7 days of antibiotic, blood culture revealed: growth organism of Staphylococcus epidermidis and from the septic marker evaluation revealed: the leukocyte count was $11.3 \mathrm{~K} / \mathrm{uL}$, platelet count was $144 \mathrm{~K} / \mathrm{uL}$, IT ratio was 0.47 . The antibiotic was changed into Cefoperazone sulbactam and Amikacin. Twenty days after surgery, the patient was lethargy and showed temperature instability. The laboratories finding revealed: leukocyte count was $22.7 \mathrm{~K} / \mathrm{uL}$, the haemoglobin level was $9.5 \mathrm{~g} / \mathrm{dL}$, platelet count was $10.16 \mathrm{~K} / \mathrm{uL}$, IT ratio was 0.06 , CRP was $165.9 \mathrm{mg} / \mathrm{dL}$. The second blood culture revealed: growth organism of Stenotrophomonas maltophilia. Based on blood culture, Amikacin and cefoperazone sulbactam changed to intravenous Levofloxacin $25 \mathrm{mg}$ every 12 hours. The patient was also given packed red cells and thrombocyte concentrate transfusion. Patient's condition was getting worse and she was passed away on the 29th day after surgery due to severe sepsis.

\section{Second Case}

A male newborn delivered at 38 weeks' gestation with spontaneous labour. He was admitted to the neonatology ward with a working diagnosis of duodenal atresia. Mother was referred due to suspicion of congenital anomaly. Antenatal care was performed on obstetrician every 3 months and the ultrasound revealed polyhydramnios on the third trimester. The baby was born vigorously with BW 2800 grams, body length of $49 \mathrm{~cm}$, and term in gestational age.

Vomiting appeared within a few hours after breastfeeding trial. The vomit was profuse and projectile, 4-6 times daily with bile-stained vomits, but without blood, the volume about $5-10 \mathrm{ml}$ of each vomits. He had mild abdominal distention and failed to pass meconium in the first 24 hours.

On the first day of life, after getting bile-stained vomit, physical examination revealed an alert and stable newborn. There was mild epigastric distention with normal skin elasticity and bowel sound was decreased. Abdominal X-ray was performed, showing gastric dilatation, no double bubble sign, but there was no gas revealed in the pelvic floor. The conclusion was suspected of duodenal atresia. Pediatric surgeon evaluated that the patient suffered a small intestinal obstruction, suspect jejunal atresia. The patient was planned to have an explorative laparotomy. 


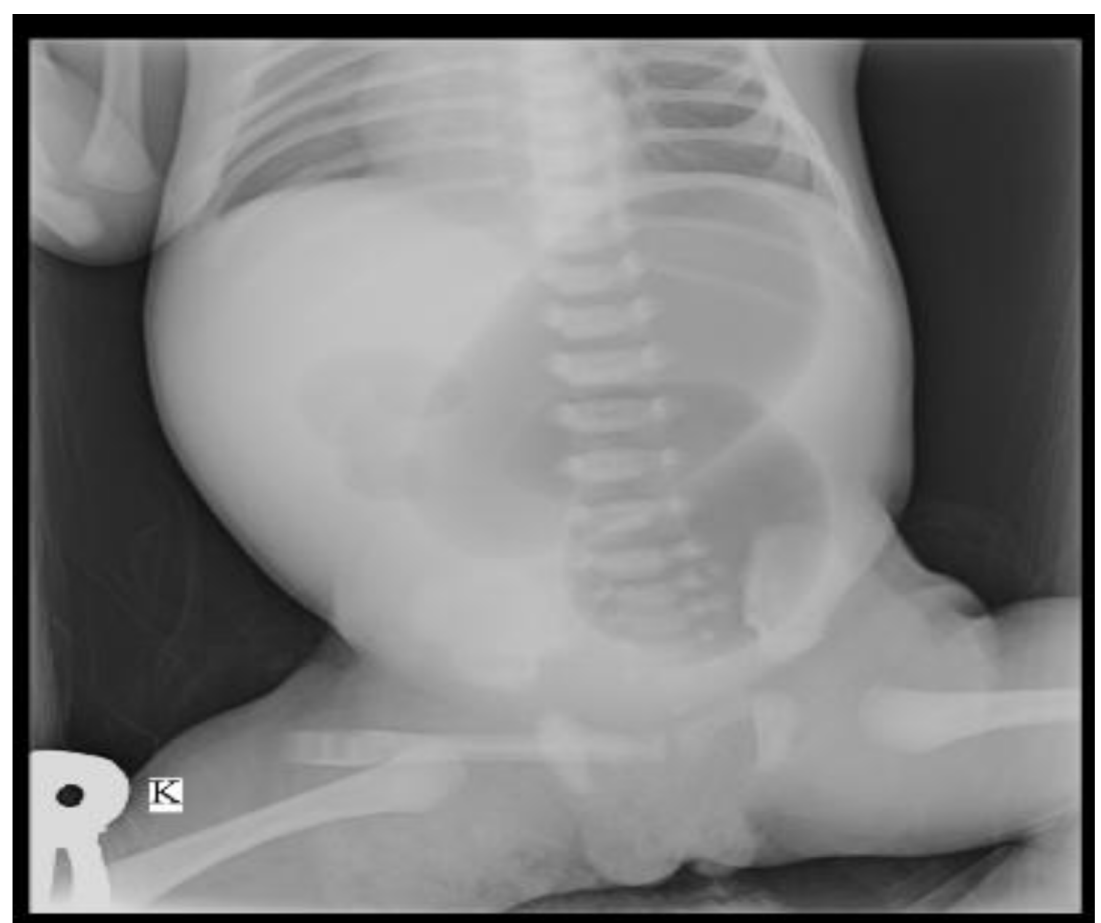

Figure 3 Abdominal X-Ray of a patient with showing dilatation of the bowel with triple bubble sign
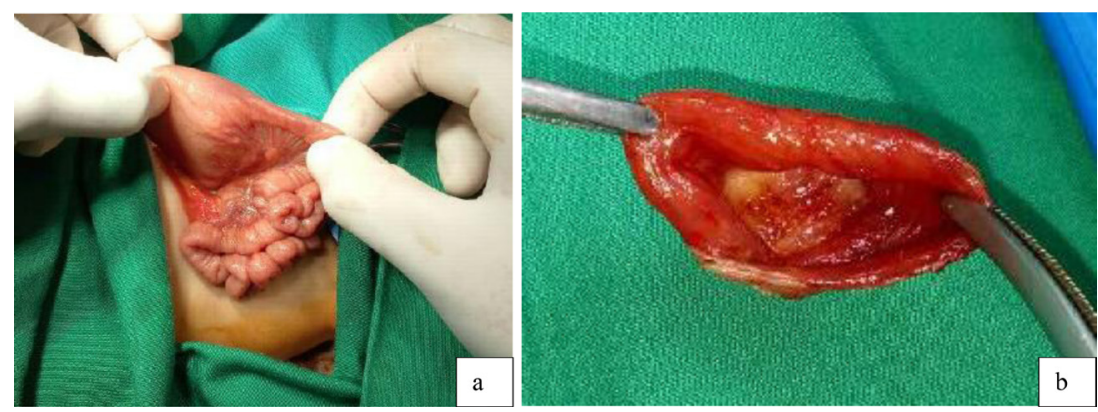

Figure 4 (a) Type I jejuno-ileal atresia with duodenum and proximal jejunum dilatation; (b) end-to-end anastomoses procedure

On the 4th day of treatment, the patient was having laparotomy. On the surgery site, a surgeon found duodenum and proximal jejunum dilatation with jejuno-ileal atresia (Figure 2a) with type IIIa. The patient had resection on jejuno-ileal atresia and continued with end to end anastomosis (Figure 2b).

The patient was treated in NICU after surgery, given total parenteral nutrition and intravenous metronidazole. In addition, the patient appeared jaundiced and was given phototherapy for 3 days. The septic marker evaluation revealed an increased IT ratio of 0.5 and procalcitonin $24.09 \mathrm{ng} / \mathrm{ml}$. The patient was suspected of early-onset neonatal sepsis and given antibiotic therapy of intravenous ampicillin and amikacin. After 7 days of antibiotic, blood culture revealed: growth organism of Enterobacter gergoviae and from the antibiotic sensitivity test, cefoperazone sulbactam was sensitive to the organism. The patient was lethargy, jaundice, and showed temperature instability, laboratories finding revealed: leukocyte $27.17 \mathrm{~K} / \mathrm{uL}$, haemoglobin $8.72 \mathrm{~g} / \mathrm{dL}$, platelet $20.67 \mathrm{~K} / \mathrm{uL}$, IT ratio 0.2 , procalcitonin $29.87 \mathrm{ng} /$ $\mathrm{ml}$. Antibiotic then changed to cefoperazone sulbactam and amikacin. The patient was also given packed red cells and thrombocyte concentrate transfusion.

Second blood culture revealed growth organism of Serratia marcescens and from the antibiotic sensitivity test, cefoperazone sulbactam was still sensitive. Antibiotic therapy was continued. The patient condition was getting worse. At the age of 19 days, the patient died due to sepsis.

\section{Third Case}

A 3-days old female newborn was referred from a private hospital with diagnosis observation of vomiting with suspicion of pyloric atresia. Vomiting appeared on the second day of birth. The vomit was green, profuse and projectile, 6-8 times daily, with volume approximately $5-10 \mathrm{ml}$ of each vomit. The first defecation was on the 3rd day of life. The baby was not doing well and has started to become yellowish since the morning.

She was born at 37-38 weeks of gestation with cesarean section because of premature rupture of membrane for more than 24 hours. No abnormality was found during antenatal care. There was no illness history during the pregnancy period noted, nor a history of consuming any medicine as well. There was also no history of congenital anomalies in the family. The patient was the third child of the family. She was born vigorously with a bodyweight of 2500 grams, body length of $48 \mathrm{~cm}$, and term in gestational age.

At the pediatric emergency unit, the baby had weak activity and muscle tone. There was no abdominal distention with normal skin elasticity; bowel sound was normal. On the skin examination, the baby appeared jaundice with icterus Kramer IV-V. An abdominal X-ray was performed (Figure 3), showing dilatation of the bowel with a triple bubble sign suggesting of jejunal atresia.

The patient was diagnosed with aterm neonate, appropriate for gestational age, suspected early-onset neonatal sepsis, observation of vomiting suspected pyloric stenosis and icterus neonatorum et causa suspect sepsis. The patient was treated at the neonatal ward with an incubator. She was fasted with OGT decompression, given total parenteral nutrition, intravenous antibiotic ampicillin and amikacin. The patient was planned to have phototherapy for $3 \times$ 24 hours and consulted to the pediatric surgeon.

Pediatric surgeon consultation results in the reassessment of a patient with jejunal atresia. The patient was planned for laparotomy exploration and jejunal resection continued with end-to-end anastomosis. On surgery site surgeon found duodenum and proximal jejunum dilatation with jejunal 
atresia with type I (Figure 4a). The patient had resection on jejunal atresia $2-3 \mathrm{~cm}$ from distal jejunum and continued with end to end anastomosis (Figure $4 \mathrm{~b}$ ).

The patient was treated at NICU after surgery. On the 8th day of treatment, septic marker evaluation revealed increased procalcitonin $76.8 \mathrm{ng} / \mathrm{ml}$. Blood culture showed no growth of bacteria. So, the first-line antibiotic then changed into intravenous Cefoperazone sulbactam and Amikacin, whereas Metronidazole was continued. After 7 days of second-line antibiotic, blood culture revealed no growth. Both clinical and laboratory data showed improvement. Antibiotic was continued to 14 days. Feeding was increased slowly while observing tolerance, signs of intestinal obstruction or anastomosis leak. On the 21 st day of treatment, the patient has reached full oral feeding, in good clinical condition. She was then discharged from the hospital with a plan for outpatient follow-up.

\section{DISCUSSION}

Intestinal atresia is a frequent cause of neonatal bowel obstruction, with multiple intestinal atresias being reported in $6-12 \%$ of cases. 1 Jejunal atresia with Type IIIA atresia is one of the rare and most severe forms of intestinal atresia. Type IIIA atresia is a serious congenital anomaly that affects approximately 1:5,000-10,000 live births. ${ }^{78}$ This type constituted $5-10 \%$ of all small bowel atresia and was first reported by Santulli and Blanc, in 1961. A mesenteric (superior mesenteric artery) ischemic insult to the developing midgut during fetal development after the 11th week of gestation is widely accepted to result in jejuno-ileal atresia, as described by Louw and Barnard from their experimental studies. ${ }^{910}$ In Type IIIA atresia, it is speculated that vascular occlusions occur in the superior mesenteric artery distal to the origin of the right colic or the ileocolic artery, in the earlier stage of gestation, then ischaemic and necrosis of the distal superior mesenteric artery occurs. The intestine elongates more readily than its artery, resulting in the helical configuration of ileum. ${ }^{11}$

Jejuno-ileal atresia usually occurs in male and term infants with weight at presentation more than 2000 grams..$^{12}$ In this case, 2 of 3 patient are term infants. Only 1 was born preterm at 34 weeks of gestation. All three cases with weight more than 2000 grams. Antenatal Ultrasonography and Magnetic Resonance Imaging (MRI) can predict intestinal atresia by the presence of dilated bowel and polyhydramnios. Polyhydramnios, an excessive accumulation of amniotic fluid within the amniotic cavity, is not associated with an identifiable cause in the majority of the cases. However, in about one-third of the cases, an underlying cause is present. ${ }^{13}$ In this case series, antenatal ultrasonography showed polyhydramnios on 2 out of 3 cases, while the last case showed no abnormality.

Almost all cases, the symptoms are presented at the age of fewer than 7 days. ${ }^{12}$ Abdominal distention and bilious vomiting are cardinal clinical findings of neonatal intestinal obstruction that may necessitate urgent operative intervention. Other manifestations include jaundice present in one-third of the infants and failure to pass meconium in the first 24 hours. ${ }^{14}$ In this case series, all cases were diagnosed within the first day of life with the symptom of bilious vomiting and mild abdominal distention. The patient also was unable to pass meconium in the early 24 hours and had jaundice.

On plain X-ray, the gas shadows can be seen up to the level of atresia with proximal bowel dilatation. ${ }^{15}$ The "triple bubble" sign is usually demonstrable on the erect plain abdominal radiograph after the first 4 hours of life. The appearance is caused by a distended and gas-filled stomach and proximal jejunum. This is unlike ileal atresia where the bubbles are more in number, and the dilated intestinal loops may be challenging to differentiate from the ahaustral colon of the neonate. Contrast studies are usually not necessary and may be associated with aspiration if attempted. Distal bowel gas indicates stenosis or incomplete membrane. ${ }^{16}$ In this case series, 2 out of 3 cases the plain abdominal radiograph showed gastric dilatation, no double or triple bubble sign, but there wasn't gas revealed in the pelvic floor. In addition, the third case showed the classical triple bubble sign even though the contrast radiography was not performed in this case.

Many operative techniques have been described for the correction of intestinal atresias, such as resection with tapering enterostomy and anastomosis, resection with enterostomy or only enterostomy, and commonly by primary resection, with end-to-oblique anastomosis. The current surgery performed is excision of the dilated proximal intestine and primary anastomosis. ${ }^{17}$ In this case series, the intraoperative findings revealed jejuno-ileal atresia with a dilated proximal jejunum and small-calibre ileum. The proximal, dilated jejunum was partially resected, and end-to-end anastomosis was done in a single extra mucosal layer.

Until recently, neonatal mortality rates with Type IIIA atresia were very high though it has come down considerably in most of the reported series in the last two decades. The most important cause of mortality remains short bowel syndrome, encountered in $65 \%$ of infants with extensive atresia, types IIIA or type IV. The other cause of mortality is due to the associated anomalies, anastomotic leak, 
functional obstruction, sepsis, and prematurity. Besides, the respiratory complications, malnutrition, and total parenteral nutrition were also related to the complications during the post-operative course. ${ }^{9,17}$ In this case, series, the first two patient's condition was getting worse and died; they were the type IV and type IIIA jejuno-ileal atresia. All patients were given total parenteral nutrition. There was no anastomotic leak, respiratory complications, and malnutrition. A study by Ekwunife et al. in Nigeria found mortality rate was more significant in types IIIA and IV jejuno-ileal atresias. ${ }^{18}$ Mortality is higher in complex atresia, which most times will require neonatal intensive care and parenteral nutrition facilities.

\section{CONCLUSION}

This is a case series of jejunal atresia in newborn. We report three cases of jejunal atresia consists of different types: type IV (first case), type IIIa (second case) and type I (third case). Cases were neonates born with signs of upper gut obstruction. Bile-stained vomiting was reported a few hours after birth and failed to pass meconium in the first 24 hours. Physical examination revealed mild abdominal distention. Postnatal abdominal X-ray of the first two cases showed dilatation of the gaster, no gas present in the pelvic floor. The third case showed triple bubble sign on abdominal $\mathrm{x}$-ray. Laparotomy revealed the type of jejuno-ileal atresia then resection on the atresias and end to end anastomosis was performed. Cases were referred to neonatal intensive care unit post-surgery with a closely monitored of fluid balance, temperature and sign of sepsis. They were given a broad spectrum of antibiotics and total parenteral nutrition. The first two cases with type IV and type IIIA died due to sepsis, while the third case with type I jejunal atresia survived.

\section{CONFLICT OF INTEREST}

None

\section{ETHICS CONSIDERATION}

Ethics approval has been obtained from the Ethics Committee, Faculty of Medicine, Universitas Udayana, Bali, Indonesia prior to the study being conducted.

\section{FUNDING}

All of authors are responsible for the funding of the study without involvement of grant, scholarship, or any other resources of funding.

\section{AUTHOR CONTRIBUTIONS}

The authors are equally contributed to the study from the conceptual framework, data gathering, data analysis, until interpreting the outcome of the case study.

\section{REFERENCES}

1. Balanescu R, Topor L, Stoica I, Moga A. Associated type IIIB and type IV multiple intestinal atresia in a pediatric patient. Chirurgia (Bucur). 2013;108(3):407-410.

2. Sirelkhatim EE, Mohamadain AA, Hamza AA. Small Bowel Atresia in Khartoum Teaching Hospital. Global Journal of Medical Research. 2013;13(6):1-5.

3. Korkmaz M, Buyukunal SC. Proximal Jejunal Atresia Consist of Two Membranous Septa. The Medical Journal of Kocatepe. 2011;12(2):101-4.

4. Khalaf AA, Al-Obaidy MA, Khalaf KF. Jejunoileal Atresia A study of 60 cases in children welfare teaching hospital. J Fac Med Baghdad. 2010;52(3):1-4.

5. Akinola RA, Osuoji RL. Apple Peel Small Bowel, A Review of Four Cases: Surgical and Radiographic Aspects. J Nepal Paediatr Soc. 2011;31(3):227-34.

6. Amole AOD, Johnson AW, Adesiyum OAM. The diagnostic value of the triple bubble sign in proximal jejunal atresia: a case report. Afr J Med Sci. 2003;32(1):95-8.

7. Schwartzberg D, Burjonrappa SC. Pseudo Double Bubble: Jejunal Duplication Mimicking Duodenal Atresia on Prenatal Ultrasound. Journal of Neonatal Surgery. 2013;2(4):1-4.

8. Machmouchi M. New successful one-step surgical repair for apple peel atresia. Open Access Surgery. 2011;4:53-6.

9. Kurbet SB, Patil HS, Dhaded SM, Bhandankar MR, Mane RS. Apple peel jejunal atresia: Successful management of a rare case. Journal of the Scientific Society. 2012;39(1):29-31.

10. Louw $\mathrm{JH}$, Barnard $\mathrm{CN}$. Congenital intestinal atresia: Observations on its origin. Lancet. 1955;269(6899):1065-7.

11. Kshirsagar AY, Desai SR, Nagvekar MA, Vasisth GOP. Apple-peel atresia presenting as foetal intestinal obstruction. African Journal of Paediatric Surgery. 2011;8(2):235-6.

12. Shahjahan M, Ferdous KMN, Mitul MAR, Islam MK. Management of Jejunoileal Atresia: Our 5 year Experience. Chattagram Maa-O-Shishu Hospital Medical College Journal. 2013;12(3):52-5.

13. Sondheimer JM. Gastrointestinal tract. In: Hay WW, Hayward HR, Levin MJ, Sondheimer JM, editors. Current pediatric diagnosis \& treatment. 16th ed. New York: The McGraw-Hill Companies, Inc; 2003. p. 614-92.

14. Dalla Vecchia LK, Grosfeld JL, West KW, Rescorla FJ, Scherer LR, Engum SA. Intestinal atresia and stenosis: a 25-year experience with 277 cases. Arch Surg. 1998;133(5):490-497.

15. Sahu S, Dhavala SS. Apple-Peel Syndrome A Case of Malrotation with Atresia of Proximal Small Bowel. Ind J Radiol Imag. 2006;16(2):189-90.

16. Tröbs RB, Tannapfel A. Apple peel small bowel. Imaging case book. J Perinatol. 2009;29(12):832-833.

17. Das PC, Avabratha KS. Apple Peel Jejunal Atresia in a Neonate: A Rare Cause of Intestinal Obstruction. International Journal of Biomedical Research. 2012;3(2):114-5.

18. Ekwunife OH, Oguejiofor IC, Modekwe VI, Osuigwe AN. Jejuno-ileal atresia: a 2-year preliminary study on presentation and outcome. Niger J Clin Pract. 2012;15(3):354-357.

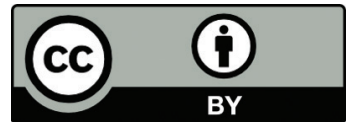

This work is licensed under a Creative Commons Attribution 